\title{
Estimation of Reduced Order Equivalent Circuit Model Parameters of Batteries from Noisy Current and Voltage Measurements
}

\author{
B. B. Alagoz, H. Alisoy
}

\begin{abstract}
Identification of reduced order equivalent circuit battery model from current and voltage measurements allows modeling, classification and monitoring of batteries, and these tasks are very essential for battery management systems. This study presents a theoretical study to investigate performance of computer-aided identification of the reduced order equivalent circuit battery model from noisy current and voltage measurement data. The battery model is expressed in the form of fractional order differential equation and time domain numerical solution of this model is numerically calculated according to Grünwald-Letnikov definition of fractional-order derivative. Paper demonstrates an application of this numerical solution so that it can fit noisy current and voltage measurement data, and thus parameters of the equivalent circuit battery model can be estimated. Particle swarm optimization (PSO) method is used to solve this model fitting problem. Performance of the parameter estimation method is investigated for various noise levels of the synthetically generated current and voltage profiles.
\end{abstract}

Index Terms - Battery model, fractional order system model, Grünwald-Letnikov definition, PSO.

\section{INTRODUCTION}

$\mathrm{D}$ UE TO growing demand for mobility in daily life, battery utilization in real applications increase and hence battery monitoring and management methods are becoming an essential component of mobile systems. In fact, practical performance of many battery powered systems e.g. mobile devices, electrical vehicles depends on battery management performance [1,2]. Nowadays, monitoring battery health and optimal control of battery packs are major topics of battery research studies, and these studies has focused on modeling and real-time identification of battery model parameters, which is useful for evaluating battery status and health [3]. Therefore, online control and the management of batteries are performed based on model parameter estimations, which can provide the meaningful information related to conditions of batteries.

B. B. ALAGOZ, is with Department of Computer Engineering, Inonu University, Malatya, Turkey, (e-mail: baykant.alagoz@inonu.edu.tr).

H. ALISOY, is with Department of Electronics and Communication Engineering, Namik Kemal University, Tekirdag, Turkey, (e-mail: halisoy@nku.edu.tr).

Manuscript received July 30, 2018; accepted October 28, 2018. DOI: $10.17694 /$ bajece.449265
There are several models that were utilized for modeling batteries in different levels of complexity [3,4]. High complexity models can be useful for more proper representation of the behavior or properties of batteries in simulation environment, however increasing model complexity can heavily complicate real-time identification problem and model parameter estimation efforts. One of the widely utilized models for battery monitoring is the reduced order equivalent circuit model. This model can be used by battery monitoring algorithms and identified either in time domain by measuring current and voltage profiles or in the frequency domain by applying impedance spectroscopy measurements [2]. Figure 1(a) shows a reduced order equivalent circuit model based on the impedance spectrum data [2,5]. Figure 1(b) illustrates an s-domain transfer function representation of this model [6]. The fractional order capacitor element makes this model a fractional order system model [6] and time-domain analysis of this model requires application of fractional calculus. Since recent developments in fractionalorder system analysis tools [7], the s-domain representation of this system can be preferable for battery parameter estimation and simulation purposes.

Grünwald-Letnikov (GL) definition of fractional order differentiation has been widely utilized for the numerical solution of fractional order differential equations, solution of fractional order system models in state space form and calculation of time response of fractional order transfer functions $[7,8,9,10,11,12]$. Numerical calculation methods based on Grünwald-Letnikov definition are commonly used to develop fractional-order system analysis tools $[7,10]$.

In previous studies, Grünwald-Letnikov based numerical solution of this battery model were utilized in many studies for estimating state of charge, parameter sensitivity analysis etc. $[1,13,14,15]$. Some of them were utilized metahereustic optimization methods, such as hybrid multi-swarm particle swarm optimization and genetic algorithm, because model fitting problem is not so easy for fractional-order systems. In addition to model coefficients (component values), the estimation of fractional-orders of the differential equation models is required, and this significantly increases complexity of numerical solution of the problem compared to fixed order differential equation models, namely integer-order system models. Main reason of this complication comes from the long memory effect, that is, fractional order derivative depends on all past values of the fractionally derived function [7] and hence fractional-order derivative is not a local operator. 

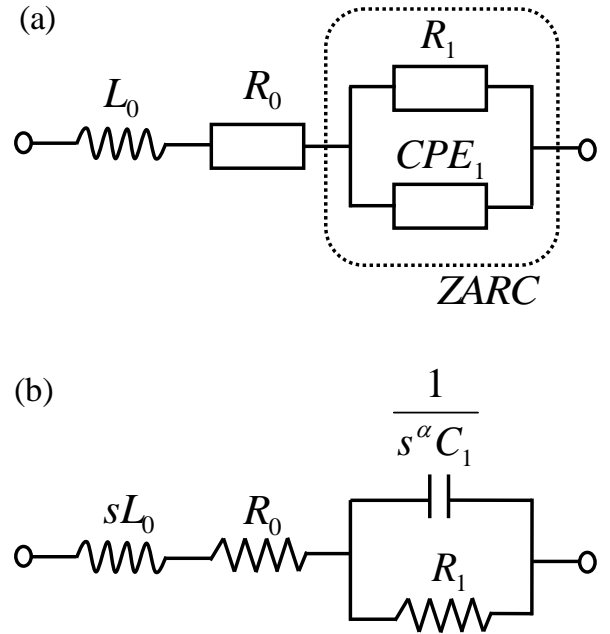

Fig. 1. (a) Reduced order equivalent circuit model [2], (b) Transfer function representation of this model in s-domain [6].

Performance of real-time model parameter estimation algorithms depends on a number of factors:

(i) Limitation of computational resources such as the speed of processor, memory capacity can decrease practical performance. Computational complexity of the estimation method should be low enough so that it can be implemented on control cards by using embedded programming techniques. (ii) Negative impacts of measurement noise on the accuracy of parameter estimation can decrease practical performance. The estimation accuracy should be enough robust against measurement noises.

(ii) For online analysis of model parameters, estimation method should be independent of waveform of the measured data because, in real applications, measured current and voltage can be in any waveforms. Real-time operating model identification algorithms should deal with waveform uncertainty of the measured data.

The current study discusses performance of a time-domain model identification approach that may be utilized for realtime estimation of parameters of the reduced order equivalent circuit model. This method performs fitting GrünwaldLetnikov based numerical solutions of the equivalent circuit model to noisy current and voltage data profiles that are measured from battery terminals. To achieve approximation of the model response to this measured data, an average of squared difference of numerical voltage and measured voltage values is used as the objective function that should be minimized to achieve identification. Here, PSO algorithm is employed to solve this optimization problem. To improve estimation of model parameters, this objective function, namely mean square error (MSE), is modified by taking logarithm of MSE function. This modification to objective function can improve minimization performance of PSO at very low values of MSEs because of the logarithmic stretching of MSE values at very low levels. Here, the logarithm of MSE can make objective function more distinguishable at very low values.

\section{PRELIMINARIES AND PROBlem StATEMENTS}

Impedance spectroscopy is a main technique that has been applied for identification of sophisticated equivalent circuit models $[2,5,16]$. This method is performed by matching real or complex parts of frequency domain models or magnitude and phase responses for the each measured frequency [2]. In this manner, the equivalent circuit model shown in Figure 1(a) is expresses in the complex impedance form,

$$
Z(\omega)=R_{o}+j \omega L_{o}+\frac{R_{1}}{1+A_{1} R_{1}(j \omega)^{\alpha}} .
$$

The last term of the equation (1) expresses a fractional order behavior, which was also known as constant phase element [2, 17]. This term is also referred to as ZARC or Cole-Cole circuit element $[17,18]$. Parameters $R_{o}$ and $L_{o}$ are output resistance and inductance of the model, respectively.

We aim to perform time domain identification of the model from a given current and voltage data set. By considering $s=j \omega$ relation, the transfer function model of the reduced order equivalent circuit model was written in the form [6],

$$
Z(s)=R_{o}+s L_{o}+\frac{R_{1}}{1+C_{1} R_{1} s^{\alpha}} .
$$

The s-domain equivalent circuit representation was shown in Figure 1(b) and this representation implies that fractional order system analysis methods can be applied to obtain time domain solutions of this model. Considering current and voltage in impedance function, by considering the equation (2), a fractional order system model is written as

$$
\begin{gathered}
V(s)=Z(s) I(s), \\
V(s)=R_{o} I(s)+s L_{o} I(s)+\frac{R_{1}}{1+C_{1} R_{1} s^{\alpha}} I(s) .
\end{gathered}
$$

To facilitate time domain solution of battery voltage, the equation (4) can be decomposed as the sum of three voltage elements as,

$$
V(s)=V_{1}(s)+V_{2}(s)+V_{3}(s),
$$

where the voltage terms are $V_{1}(s)=R_{o} I(s)$ for the voltage across $R_{o}, \quad V_{2}(s)=L_{o} s I(s)$ for the voltage across $L_{o}$ and $V_{3}(s)=\frac{R_{1}}{1+C_{1} R_{1} s^{\alpha}} I(s)$ for constant phase element. By taking inverse Laplace transform of these voltage components, time domain solution of the components can be written as follows,

$$
\begin{aligned}
& v_{1}(t)=R_{o} i(t), \\
& v_{2}(t)=L_{o} \frac{d i(t)}{d t},
\end{aligned}
$$

where $i(t)$ is the battery current. To obtain time domain model for $V_{3}(s)$, one can reorganize it as $C_{1} R_{1} s^{\alpha} V_{3}(s)+V_{3}(s)=R_{1} I(s)$, and by taking inverse Laplace transform and using the properties of $L\left\{D^{\alpha} F(s)\right\}=s^{\alpha} f(t)$, it can be expressed in time domain as,

$$
C_{1} R_{1} D^{\alpha} v_{3}(t)+v_{3}(t)=R_{1} i(t),
$$

Numerical solutions of this fractional-order differential equation can be obtained by using Grünwald-Letnikov 
definition of fractional-order derivative operator, which is written by [7],

$$
{ }_{a} D_{t}^{\alpha} f(t)=\lim _{h \rightarrow 0} \frac{1}{h^{\alpha}} \sum_{j=0}^{[(t-a) / h]} w_{j}^{(\alpha)} f(t-j h),
$$

where $a$ parameter is a lower bound for time derivative and it limits calculations from the time $a$ to current time. Although it reduces need of memory elements to store the past values, it results in a truncation error in calculations. For more accurate calculation of fractional derivatives in system analysis, $a$ can be zero. Parameter $h$ is step size (sampling period) for computations. The operator $[(t-a) / h]$ expresses the rounding the value of term $(t-a) / h$ to a nearest smaller integer number. The weight parameter $w_{j}^{(\alpha)}$ for $j=0,1,2, \ldots$ is written recursively as [7],

$$
w_{0}^{(\alpha)}=1, w_{j}^{(\alpha)}=\left(1-\frac{\alpha+1}{j}\right) w_{j-1}^{(\alpha)} .
$$

For a finite and non-zero time sampling $h$ and considering values of $f(t-j h)$ from zero to current time by taking $a=0$, one expresses an approximate formulation of GrünwaldLetnikov differentiation as,

$$
D_{t}^{\alpha} f(t) \cong \frac{1}{h^{\alpha}} \sum_{j=0}^{[t / h]} w_{j}^{(\alpha)} f(t-j h) .
$$

The equation (11) is used in equation (8), the numerical solution of this differential equation can be found by solving,

$$
C_{1} R_{1} \frac{1}{h^{\alpha}} \sum_{j=0}^{[t / h]} w_{j}^{(\alpha)} v_{3}(t-j h)+v_{3}(t) \cong R_{1} i(t) .
$$

This equation can be rewritten as

$$
C_{1} R_{1} \frac{1}{h^{\alpha}} w_{0}^{(\alpha)} v_{3}(t)+C_{1} R_{1} \frac{1}{h^{\alpha}} \sum_{j=1}^{[t / h]} w_{j}^{(\alpha)} v_{3}(t-j h)+v_{3}(t) \cong R_{1} i(t) .
$$

By rearranging the equation (13), the solution $v_{3}(t)$ can be written as,

$$
v_{3}(t) \cong \frac{-C_{1} R_{1}}{C_{1} R_{1} w_{0}^{(\alpha)}+h^{\alpha}} \sum_{j=1}^{[t / h]} w_{j}^{(\alpha)} v_{3}(t-j h)+\frac{R_{1} h^{\alpha}}{C_{1} R_{1} w_{0}^{(\alpha)}+h^{\alpha}} i(t) .
$$

The weights $w_{j}^{(\alpha)}$ is calculated recursively according to equation (10). By considering equation (5), a battery voltage solution with respect to a given current $i(t)$ can found by

$$
v(t)=v_{1}(t)+v_{2}(t)+v_{3}(t) .
$$

By considering equation (6), (7) and (14), $v_{1}(t), v_{2}(t)$ and $v_{3}(t)$ are calculated in discrete time domain for a current sampling $i_{m}(n)$ with a sampling $t=n h$ :

$$
\begin{gathered}
v_{1}(n)=R_{o} i(n) \\
v_{2}(n)=L_{o} \frac{i(n)-i(n-1)}{h} \\
v_{3}(n)=-\frac{C_{1} R_{1}}{C_{1} R_{1} w_{0}^{(\alpha)}+h^{\alpha}} \sum_{j=1}^{[t / h]} w_{j}^{(\alpha)} v_{3}(n-j)+\frac{R_{1} h^{\alpha}}{C_{1} R_{1} w_{0}^{(\alpha)}+h^{\alpha}} i(n)
\end{gathered}
$$

where $h$ is the sampling period and the battery voltage can be calculated by

$$
v_{a}(n)=v_{1}(n)+v_{2}(n)+v_{3}(n)
$$

An example Matlab code, which performs the numerical calculation of the reduced order equivalent circuit battery model is presented in Appendix section.

\section{IDENTIFICATION OF MODEL PARAMETERS}

Let's denote the measured current and voltage from battery terminals by $i_{m}(n)$ and $v_{m}(n)$ signals, respectively. For fitting the $i_{m}(n)$ and $v_{m}(n)$ data $(i=0,1,2, . ., p)$ to time domain solution of the equivalent circuit battery model, which is calculated by equation (15), a mean square error to minimize is commonly written by $[10,13-15]$

$$
E=\frac{1}{p} \sum_{n=1}^{p}\left(v_{a}(n)-v_{m}(n)\right)^{2},
$$

where, $p$ is the total number of the sampled data. The $v_{a}(n)$ is calculated according to the equation (19). To increase accuracy in the estimation of model parameters, MSE, given by equation (20), can be modified by taking logarithm of MSE function. This makes very low error values more distinguishable as a result of stretching effect of logarithmic at very low values. The logarithmic error function can be written by,

$$
E_{l}=10 \log \left(\frac{1}{p} \sum_{n=1}^{p}\left(v_{a}(n)-v_{m}(n)\right)^{2}\right) .
$$

To estimate the reduced order equivalent model parameters of battery, which are $C_{1}, R_{1}, R_{0}, L_{0}$ and $\alpha$, the optimization problem $\min _{C_{1}, R_{1}, R_{0}, L_{0}, \alpha} E_{l}$ can be solved by metaheuristic optimization methods. In this study, PSO algorithm is used to solve this optimization problem (see Figure 12 in Appendix section).

\section{Simulation StUdy}

To estimate model parameters $C_{1}, R_{1}, R_{0}, L_{0}$ and $\alpha$ according to battery terminal measurements $i_{m}(n)$ and $v_{m}(n)$, we implemented PSO algorithm to minimize $E_{l}$ by using numerical formulation that is derived in the previous section.

To evaluate parameter estimation performance of the method for noisy data, we considered Li-ion battery parameters that were identified as $C_{1}=72 \mathrm{~F}, R_{1}=4.17 \mathrm{~m} \Omega$, $R_{0}=0.71 \mathrm{~m} \Omega, \quad L_{0}=3.5110^{-7} \mathrm{H}$ and $\alpha=0.72$ in [2]. To generate synthetic noise at various SNR levels, a random noise signal is added to the ground truth current data $i(n)$ and voltage data $v(n)$. The ground truth terminal voltage $v(n)$ is calculated for a terminal current waveform $i(n)$ by using equation (19). The synthetic noisy test data were generated as,

$$
\begin{aligned}
& i_{m}(n)=i(n)+\eta_{i}(n), \\
& v_{m}(n)=v(n)+\eta_{v}(n),
\end{aligned}
$$

where $\eta_{i}$ is random noise signal for current measurements and $\eta_{v}$ is random noise signal for voltage measurements. PSO algorithm was applied for the parameter search ranges of $C_{1} \in[0,200] \quad \mathrm{F}, \quad R_{1} \in(0,20) \quad \mathrm{m} \Omega, \quad R_{0} \in[0,10] \mathrm{m} \Omega$, $L_{0} \in\left[0,10^{-6}\right] \mathrm{H}$ and $\alpha \in[0,2]$. Population size of PSO was set to 30 particles and the maximum iteration number was configured to 1200 iterations. Simulation and test environment were developed in Matlab program. 
Firstly, noise signals $\eta_{i}$ and $\eta_{v}$ were set to zero for noisefree test and model parameter estimation for this noise-free case was performed to verify operation of the algorithm. PSO yielded estimations of $C_{1} \cong 72.00 \quad \mathrm{~F} \quad, R_{1} \cong 4.17 \mathrm{~m} \Omega$, $R_{0} \cong 0.71 \mathrm{~m} \Omega, L_{0} \cong 3.5110^{-7} \mathrm{H}$ and $\alpha \cong 0.72$, which are the almost exact values of ground truth (original) data of Li-ion battery model. Logarithmic MSE was obtained $E_{l}=-196.88$, which corresponds to $E=2.194810^{-18}$, that is, the error is almost zero. Figure 2 shows accurately fitting of the model voltage $v_{a}(n)$ and the voltage data $v_{m}(n)$ for this zero-noise case. To better view plots, $v_{m}(n)$ is drawn subsampled in the figure.

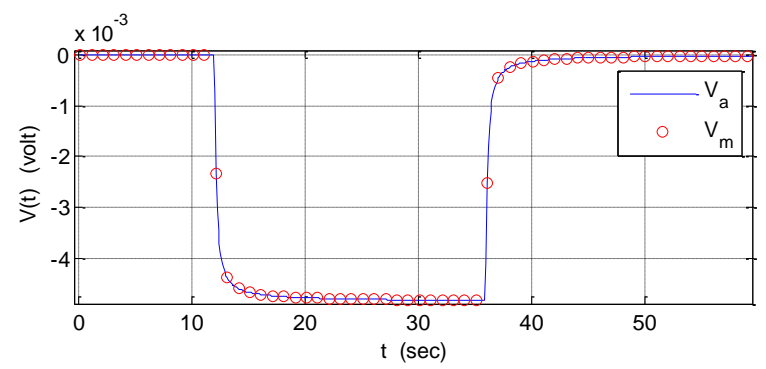

Fig. 2. Fitting of the model voltage $v_{a}(n)$ and the voltage data $v_{m}(n)$ for zero-noise case $v_{m}(n)$ data is shown subsampled in the figure.

For a low-level noisy measurement scenario, the current waveform was noised at $S N R=59.49 \mathrm{~dB}$ and ground truth voltage was noised at $S N R=48.16 \mathrm{~dB}$. Figure 3 shows a satisfactory fitting of the model voltage $v_{a}(n)$ and the voltage data $v_{m}(n)$ for these noise data. After fitting by PSO algorithm, the model parameters were estimated $C_{1} \cong 72.04 \mathrm{~F}$ $, R_{1} \cong 4.16 \mathrm{~m} \Omega, \quad R_{0} \cong 0.72 \quad \mathrm{~m} \Omega, \quad L_{0} \cong 5.1210^{-7} \mathrm{H} \quad$ and $\alpha \cong 0.716$, which are the slightly different from ground truth values of parameters. Logarithmic MSE was obtained $E_{l}=-91.01$, which corresponds to $E=8.3910^{-10}$. This level of MSE is acceptable for practical performance.



Fig. 3. Fitting of the model voltage $v_{a}(n)$ and the voltage data $v_{m}(n)$ for low-level noise current ( $S N R=59.49 \mathrm{~dB}$ ) and low-level noise voltage data ( $S N R=48.16 \mathrm{~dB}) . v_{m}(n)$ data is shown subsampled in the figure.

For a mid-level noisy measurement scenario, the current waveform was noised at $S N R=41.08 \mathrm{~dB}$ level and the ground truth voltage was noised at $S N R=32.45 \mathrm{~dB}$. Figure 4 shows noisy current and noisy voltage data. Figure 5 demonstrates fitting performance of the $v_{a}(n)$ and the $v_{m}(n)$. After performing PSO, the model parameters were estimated as $C_{1} \cong 74.31 \mathrm{~F}, R_{1} \cong 4.24 \mathrm{~m} \Omega, R_{0} \cong 0.64 \mathrm{~m} \Omega, L_{0} \cong 110^{-6} \mathrm{H}$ and $\alpha \cong 0.714$. They are more differentiated from ground truth values of parameters compared to previous low-noise measurement test. Logarithmic MSE was obtained $E_{l}=-76.68$, which corresponds to MSE of $E=2.3010^{-8}$. This accuracy level can be acceptable for some battery parameter estimation applications.
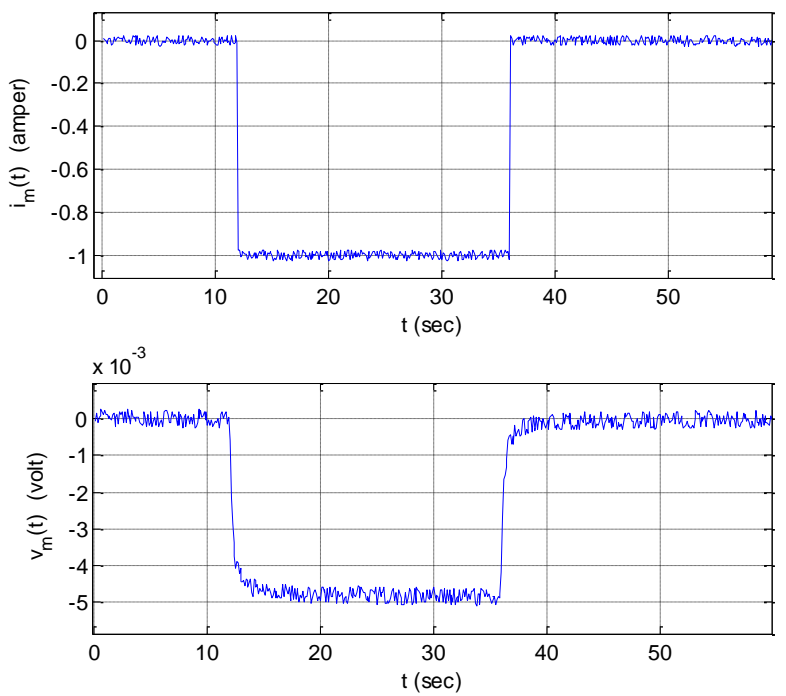

Fig. 4. Low-level noisy current data $(S N R=41.08 \mathrm{~dB})$ and mid-level noisy voltage data $(S N R=32.45 \mathrm{~dB})$

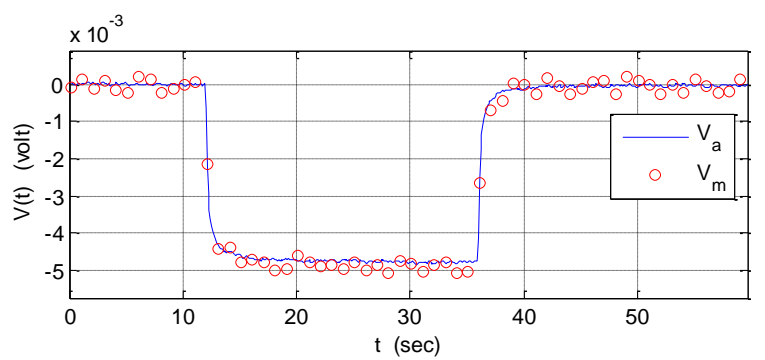

Figure 5. Fitting of the model voltage $v_{a}(n)$ and the voltage data $v_{m}(n)$ for mid-level noise current ( $S N R=41.08 \mathrm{~dB}$ ) and mid-low noise voltage data $(S N R=32.45 \mathrm{~dB}) . v_{m}(n)$ data is shown subsampled in the figure.

For high-level noisy measurement scenario, the current waveform was noised at $S N R=36.22 \mathrm{~dB}$ level and the ground truth voltage was noised at $S N R=28.46 \mathrm{~dB}$ in Figure 6 . Figure 7 shows the fitting of the model voltage $v_{a}(n)$ to the $v_{m}(n)$ under this noise levels. PSO algorithm found the model parameter as $C_{1} \cong 77.14 \mathrm{~F}, R_{1} \cong 4.06 \mathrm{~m} \Omega, R_{0} \cong 0.81 \mathrm{~m} \Omega$, $L_{0} \cong 110^{-6} \mathrm{H}$ and $\alpha \cong 0.762$. Logarithmic MSE was obtained $E_{l}=-70.66$, which corresponds to MSE of $E=8.9210^{-8}$. This level of accuracy can be adequate for insensitive applications of battery parameter estimation. 

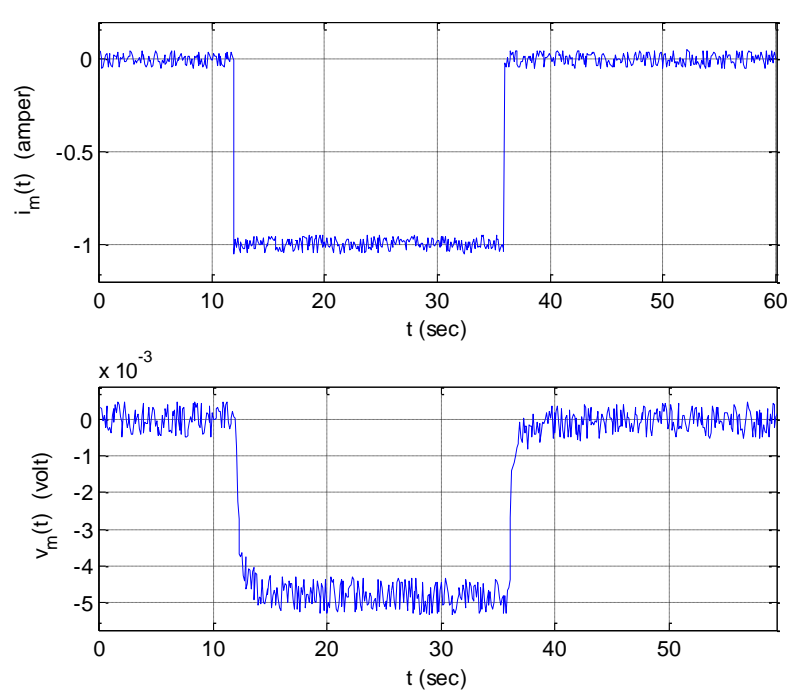

Fig. 6. High-level noisy current data ( $S N R=36.22 \mathrm{~dB}$ ) and highly noisy voltage data $(S N R=28.46 \mathrm{~dB})$

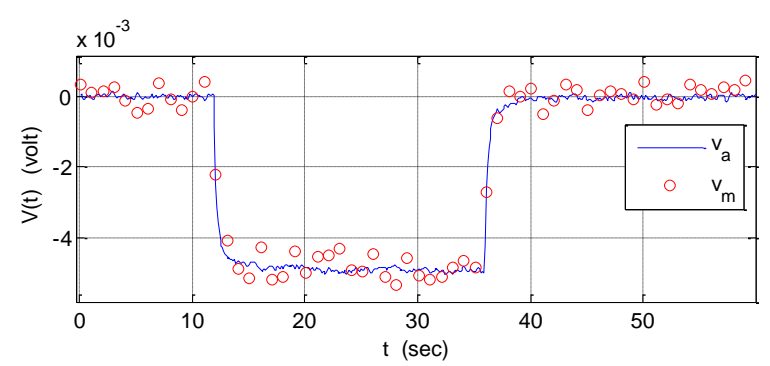

Fig. 7. Fitting of model the voltage $v_{a}(n)$ and the voltage data $v_{m}(n)$ for high-level noise current ( $S N R=36.22 \mathrm{~dB}$ ) and high-level noise voltage data $(S N R=28.46 \mathrm{~dB}) . v_{m}(n)$ data is shown subsampled in the figure.

To test algorithm for a severe noisy measurement case, the current waveform was noised at $S N R=30.05 \mathrm{~dB}$ level and ground truth voltage waveform was noised at $S N R=7.59 \mathrm{~dB}$ as illustrated in Figure 8. Figure 9 shows the fitting of the model voltage $v_{a}(n)$ and the voltage data $v_{m}(n)$. PSO algorithm estimated the model parameter as $C_{1} \cong 114.01 \mathrm{~F}$ $, R_{1} \cong 4.31 \mathrm{~m} \Omega, R_{0} \cong 0.83 \mathrm{~m} \Omega, L_{0} \cong 110^{-6} \mathrm{H}$ and $\alpha \cong 0.869$, which may not be enough accurate for sensitive battery management applications such as precise battery control. Inaccuracy of model parameters can cause misleading of controller tuning, and it decreases control system performance. Logarithmic MSE was obtained $E_{l}=-50.79$, which corresponds to a MSE of $E=8.3510^{-6}$. However, fitting of model voltage $v_{a}(n)$ and the voltage data $v_{m}(n)$ may be acceptable for some insensitive battery applications.

Table 1 summarizes results of parameter estimations that were obtained in the analyses. The absolute relative errors for parameter estimations were calculated by $e_{r}=\left|x_{e}-x_{o}\right| / x_{o}$, where $x_{e}$ stands for value of estimated parameter and $x_{o}$ is its original (ground truth) value.
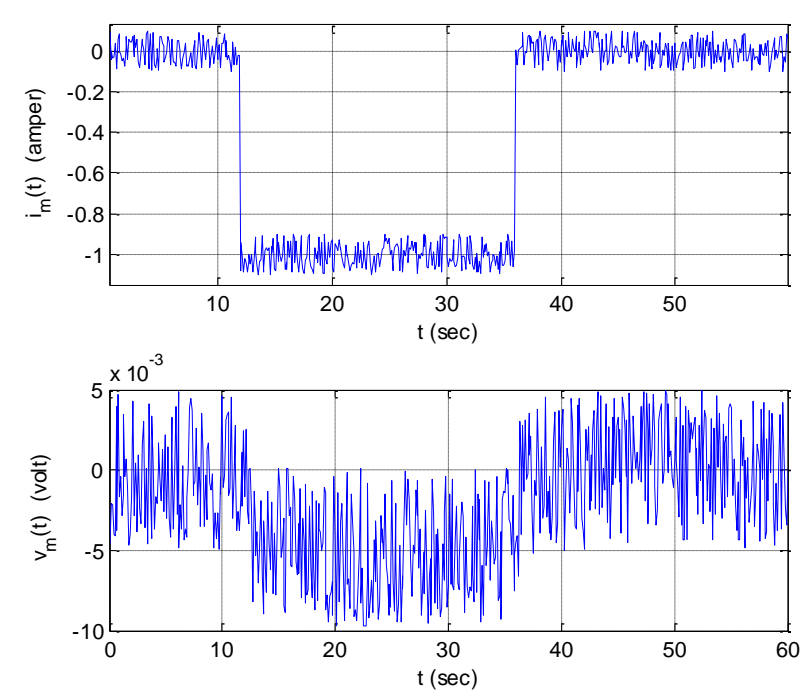

Fig. 8. Severely noised current data ( $S N R=30.05 \mathrm{~dB}$ ) and voltage data $(S N R=7.59 \mathrm{~dB})$

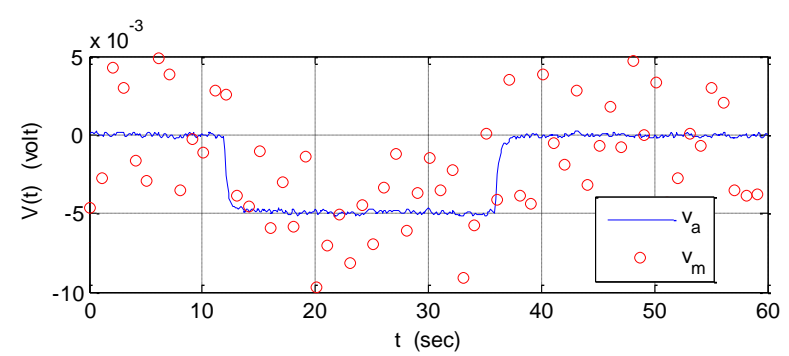

Fig. 9. Fitting of the model voltage $v_{a}(n)$ and the voltage data $v_{m}(n)$ for severely noised current $(S N R=30.05 \mathrm{~dB})$ and voltage data $(S N R=7.59 \mathrm{~dB}) . v_{m}(n)$ data is shown subsampled in the figure.

The performance data in Table 1 reveals that the estimation of resistance values, $R_{1}$ and $R_{0}$, are more robust against noise interference than those of $C_{1}$ and $L_{0}$. Main reason is that, $R_{1}$ and $R_{0}$ are static elements that are mainly effective in determining DC level of the measured signal. Smoothing effect of fitting operation can reduce negative impacts of irregular pattern of noise on the estimation of $R_{1}$ and $R_{0}$, and this makes their estimations more robust against random noise signal. The dynamic components $C_{1}$ and $L_{0}$ mostly act on transient parts of the measured signal. Therefore, irregular motif of noise can easily interfere predictions of these parameters, and this may reduce accuracy of estimations of parameters $C_{1}$ and $L_{0}$. In particular, since $L_{0}$ parameter is directly associated with derivative of the measured current, $L_{0}$ estimations are very sensitive to the noise of current measurements. Therefore, in case of noisy data, parameter search range of $L_{0}$ should be confined to its realistic ranges in order to avoid misleading parameter estimation results. In our analyses, we restricted $L_{0} \in\left[0,10^{-6}\right]$ in PSO. The $10^{-6}$ values for $L_{0}$ in Table 1 are mainly results of this restriction. 
TABLE I

A LIST OF PARAMETER ESTIMATIONS FOR VARIOUS NOISE CONDITIONS AND ABSOLUTE RELATIVE ERRORS IN PARAMETER ESTIMATION

\begin{tabular}{|c|c|c|c|c|c|c|}
\hline Parameters & $\begin{array}{c}\text { Original } \\
\text { Values } \\
\left(x_{o}\right)\end{array}$ & $\begin{array}{c}\text { Zero Noise } \\
\text { Case } \\
\left(x_{e}, e_{r}\right)\end{array}$ & $\begin{array}{c}\text { Low-level Noise } \\
\text { Case } \\
\left(x_{e}, e_{r}\right)\end{array}$ & $\begin{array}{c}\text { Mid-level } \\
\text { Noise Case } \\
\left(x_{e}, e_{r}\right)\end{array}$ & $\begin{array}{c}\text { High Level } \\
\text { Noise Case } \\
\left(x_{e}, e_{r}\right)\end{array}$ & $\begin{array}{c}\text { Severe Noise } \\
\text { Case } \\
\left(x_{e}, e_{r}\right)\end{array}$ \\
\hline$C_{1}$ & 72.00 & $72.00,0 \%$ & $72.04,0.05 \%$ & $74.31,3.2 \%$ & $77.14,7.1 \%$ & $114.01,58 \%$ \\
\hline$R_{1}$ & 4.17 & $4.17,0 \%$ & $4.16,0.2 \%$ & $4.24,1.6 \%$ & $4.06,2.6 \%$ & $4.31,3.3 \%$ \\
\hline$\alpha$ & 0.72 & $0.72,0 \%$ & $0.716,0.5 \%$ & $0.714,0.8 \%$ & $0.762,5.8 \%$ & $0.869,20.6 \%$ \\
\hline$R_{0}$ & 0.71 & $0.71,0 \%$ & $0.72,1.4 \%$ & $0.64,9.8 \%$ & $0.81,14 \%$ & $0.83,16.9 \%$ \\
\hline$L_{0}$ & $3.5110^{-7}$ & $3.5110^{-7}, 0 \%$ & $5.1210^{-7}, 45.8 \%$ & $10^{-6}, 184 \%$ & $10^{-6}, 184 \%$ & $10^{-6}, 184 \%$ \\
\hline $\operatorname{MSE}(E)$ & - & $2.1910^{-18}$ & $8.3910^{-10}$ & $2.3010^{-8}$ & $8.9210^{-8}$ & $8.3510^{-6}$ \\
\hline
\end{tabular}

Waveform of the current can affect accuracy of parameter estimations. While smooth current waveforms or steady parts of current waveforms can improve estimation of static elements, such as $R_{1}$ and $R_{0}$, whereas the altering part of waveforms can improve the estimation of dynamic elements, which are $C_{1}$ and $L_{0}$ in the model. Figure 6 shows test results for a continuous sinusoidal current waveform that was obtained by $i_{m}(t)=-2+\sin (1.25 t)+\sin (0.31 t)$ with a lowlevel noise insertion at $S N R=48.98 \mathrm{~dB}$. The voltage response $v_{m}(t)$ was obtained by the reduced order equivalent circuit model of Li-ion battery with a low-level noise insertion at $S N R=47.31 \mathrm{~dB}$. Figure 10 illustrates waveforms of the generated low-level noisy current and voltage data. Figure 11 shows fitting of output of the identified model to the measured voltage data. PSO algorithm found the model parameter as $C_{1} \cong 75.98 \mathrm{~F}, R_{1} \cong 41.05 \mathrm{~m} \Omega, R_{0} \cong 0.77 \mathrm{~m} \Omega, \quad L_{0} \cong 110^{-6} \mathrm{H}$ and $\alpha \cong 0.731$. Logarithmic MSE was obtained $E_{l}=-70.62$, which corresponds to a MSE of $E=1.0710^{-7}$. This simulation confirms that the method can be applied for any measurement waveforms, and therefore it can be used for real-time monitoring of batteries.

\section{CONCLUSION}

This study theoretically discussed performance of a scheme for online estimation of reduced order equivalent circuit model parameters of batteries from noisy and irregular waveform current and voltage measurement data. Battery model parameter estimation is needed for modeling, classification and monitoring of batteries in real applications. Temporal change of battery model parameters can be utilized for detection of battery status, diagnoses of aging effect, faults and defects. Besides, real-time identification of battery models can allow optimally control of battery charge and discharge processes. Therefore, properly estimation of battery model parameter is very essential for battery management systems and algorithms. Nowadays, increase of battery usage as a result of growing demands of electrical vehicles and mobile applications places battery modeling and parameter estimation efforts at the center of battery management system researches.
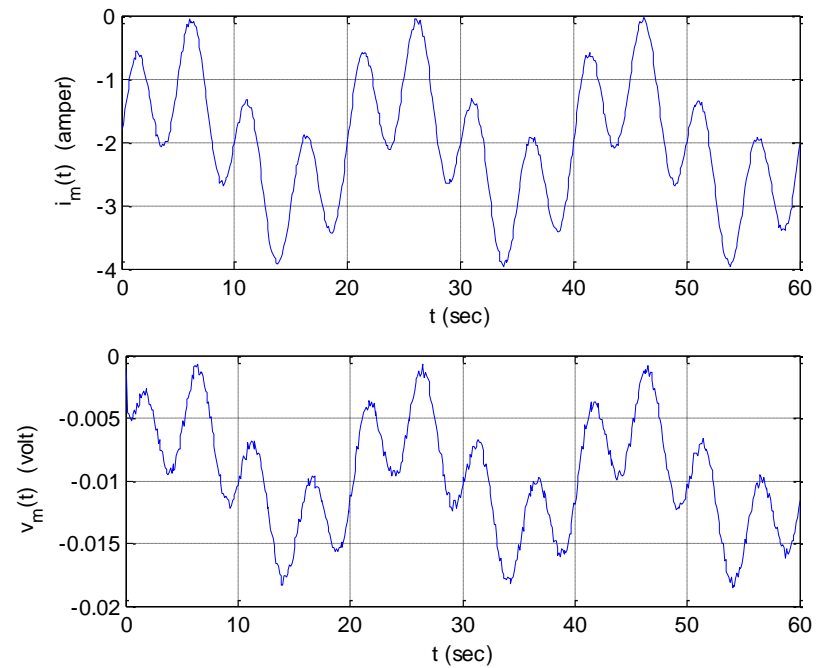

Fig. 10. Low-level noisy current data $(S N R=48.98 \mathrm{~dB})$ and low-level noisy voltage data $(S N R=47.31 \mathrm{~dB})$

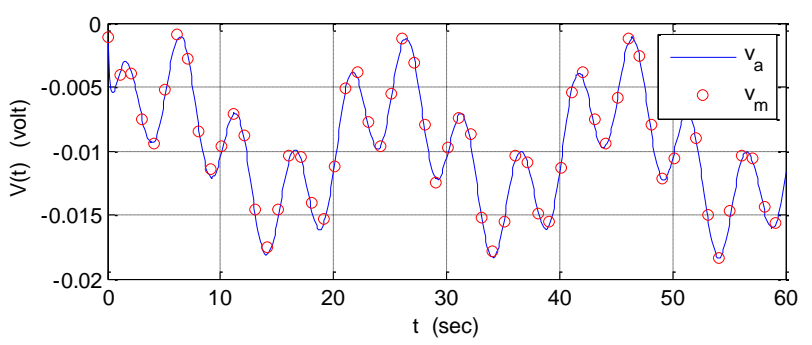

Fig. 11. Fitting of the model voltage $v_{a}(n)$ and the voltage data $v_{m}(n)$ for severely noise current ( $S N R=48.98 \mathrm{~dB}$ ) and low noise voltage data $(S N R=47.31 \mathrm{~dB}) . v_{m}(n)$ data is shown subsampled in the figure.

The presented solution for battery parameter estimation can estimates the reduced order equivalent circuit model parameters by fitting Grünwald-Letnikov based numerical solutions of the battery model to current and voltage profiles of the batteries. This fitting problem was expressed in the form of minimization of a logarithmic MSE function of battery voltages and solved by employing PSO algorithm. The numerical studies, which were carried out for various levels of 
random noise addition to input and output of Li-ion battery models, reveal that this method can work effectively in case of noisy measurements. Therefore, we conclude that the model parameter estimation of this method may be applied in real battery management applications. A future study should be conducted for experimental validation of performance of this method in experimental systems.

\section{Appendixes}

An example Matlab code to perform for numerically calculation of voltage of the reduced order equivalent circuit battery model is given, below. This code calculates battery voltage $v_{a}(n)$ for a given current waveform $i_{m}(n)$ and the battery model parameters.

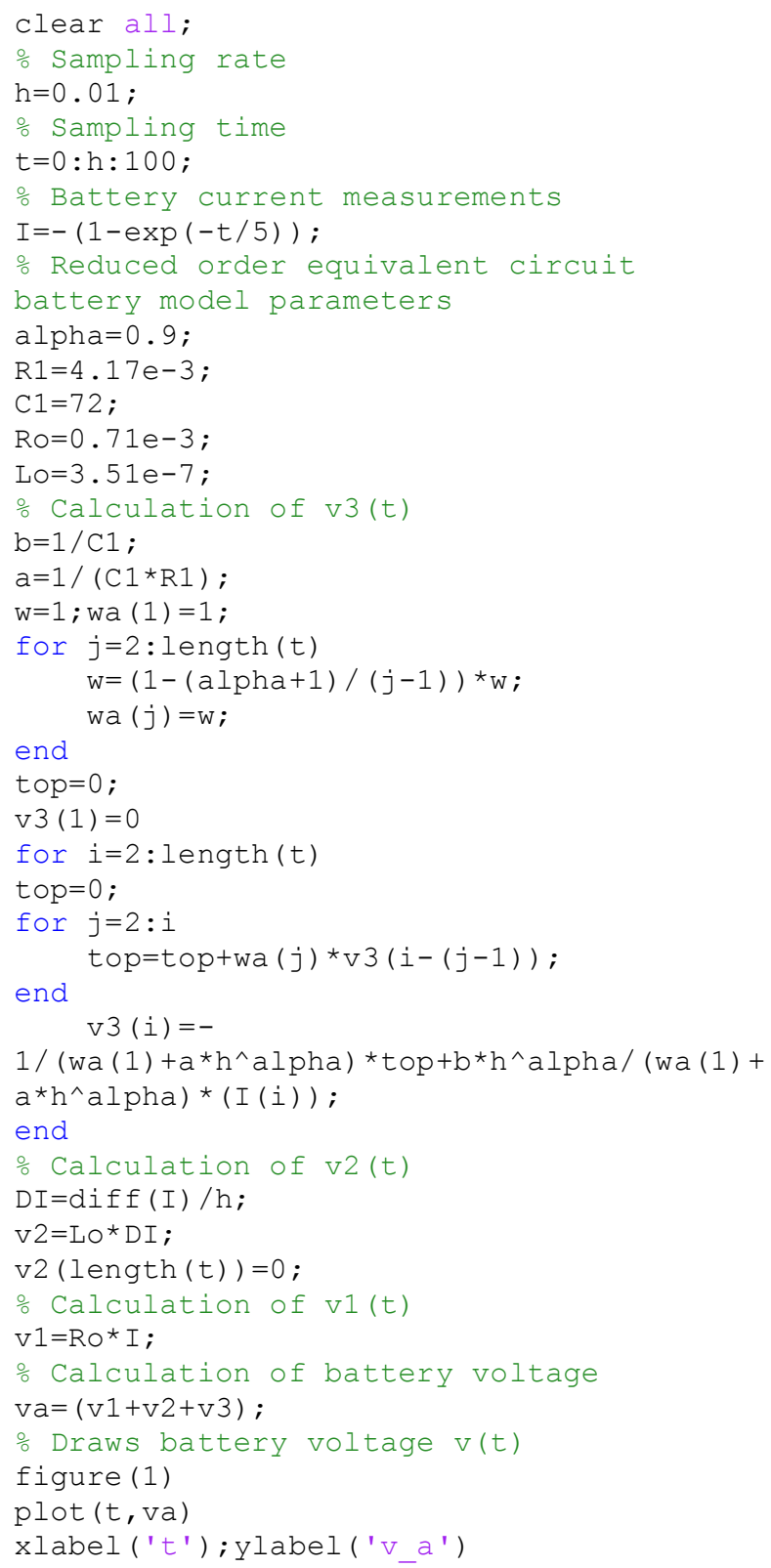

This program performs equation (19) to obtain GL solution of battery voltage. This code can be used in optimization process as shown bellow.

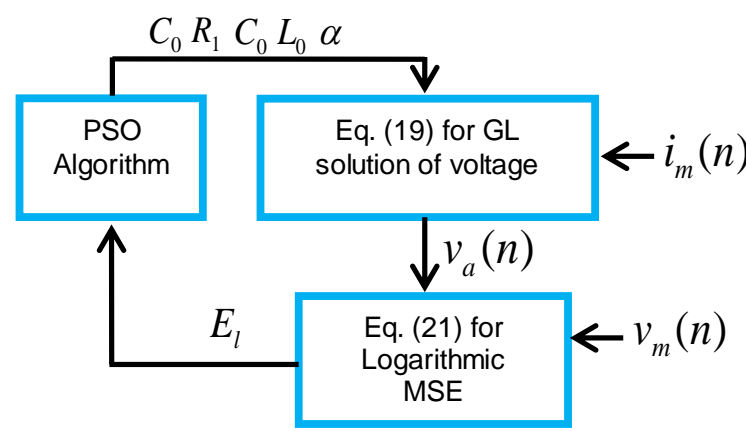

Fig. 12. A basic block diagram that shows application of PSO algorithm in this problem

\section{References}

[1] J.X. Chuntin,g C. Mi, B. Cao, J. Cao, "New method to estimate the state of charge of lithium-ion batteries based on the battery impedance model", Journal of Power Sources, Vol.233, 2013, pp.277-284.

[2] W. Waag, S. Käbitz, D.U. Sauer, "Application-specific parameterization of reduced order equivalent circuit battery models for improved accuracy at dynamic load", Measurement, Vol.46, 2013, pp. 4085-4093.

[3] C.B. Shin, BATTERIES: modeling, in: Encyclopedia of Electrochemical Power Sources, vol. I, Elsevier, 2009. pp.510-521.

[4] X. Hu, S. Li, H. Peng, "A comparative study of equivalent circuit models for Li-ion batteries", Journal of Power Sources, Vol.198, 2012, pp.359-367.

[5] S. Buller, "Impedance-Based Simulation Models for Energy Storage Devices in Advanced Automotive Power Systems", Institue for Power Electronics and Electrical Drives, RWTH Aachen University, Ph.D. Thesis, 2003.

[6] T.J. Freeborn, B. Maundy, A.S. Elwakil, "Fractional-order models of supercapacitors, batteries and fuel cells: a survey", Mater Renew Sustain Energy Vol.4, No.2, 2015, pp.1-7

[7] Y.Q. Chen, Ivo Petras, D. Xue, "Fractional order control-a tutorial", In: American Control Conference, 2009. ACC'09. IEEE, 2009. p. 13971411.

[8] R. Scherer, S.L. Kalla, Y. Tang, J. Huang, "The Grünwald-Letnikov method for fractional differential equations", Computers and Mathematics with Applications Vol.62, 2011, pp.902-917.

[9] C. Zhao, L. Luo, Y. Zhao, "Fractional Modeling Approach with MittagLeffler Functions for Linear Fractional-order System", 2012 Fifth International Conference on Intelligent Computation Technology and Automation, pp. 386- 389.

[10] R.S. Awski, K.J. Latawiec, M. Lukaniszyn, W. Czuczwara, and R. Kopka, "Modeling and identification of fractional first-order systems with Laguerre-Grunwald-Letnikov fractional-order differences", 2016 21st International Conference on Methods and Models in Automation and Robotics (MMAR),2016,pp.174-177.

[11] P. Sopasakisy, S. Ntouskas, H. Sarimveis, "Robust Model Predictive Control for Discrete-time Fractional-order Systems", 2015 23rd Mediterranean Conference on Control and Automation (MED), Torremolinos, Spain, 2015, pp. 384-389.

[12] E Dorcák, J. Valsa, J. Terpák, E. Gonzalez, "Comparison of the methods for the calculation of fractional-order differential equations", In Carpathian Control Conference (ICCC), 2011, pp. 80-84.

[13] S.E. Li, B. Wang, H. Peng, and X. Hu, "An electrochemistry-based impedance model for lithium-ion batteries," J. Power Sour., Vol. 258, 2014, pp.9-18.

[14] B. Wang, Z. Liu, S.E. Li, , S.J. Moura, H. Peng, "State-of-charge estimation for lithium-ion batteries based on a nonlinear fractional model", IEEE Transactions on Control Systems Technology, Vol.25, No.1, 2017, pp. 3-11. 
[15] D. Zhou, K. Zhang, A. Ravey, F. Gao, A. Miraoui, "Parameter sensitivity analysis for fractional-order modeling of lithium-ion batteries", Energies, Vol.9, No.3, 2016, pp.123.

[16] A. Fotouhi, D.J.Auger,K. Propp,S. Longo,M. Wild,A review on electric vehicle battery modelling: From Lithium-ion toward LithiumSulphur, Renewable and Sustainable Energy Reviews,Vol.56,2016,pp.1008-1021.

[17] E. Barsukov, J.R. Macdonald, Impedance Spectroscopy: Theory, Experiment and Applications, second ed., Wiley-Interscience, 2005.

[18] M.E. Orazem, P. Shukla, M.A. Membrino, "Extension of the measurement model approach for deconvolution of underlying distributions for impedance measurements", Electrochimica Acta, Vol.47, 2002, pp.2027-2034.

\section{BIOGRAPHIES}

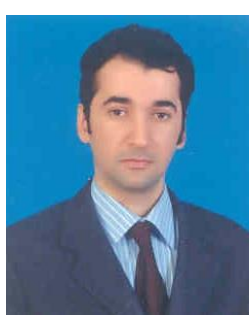

BARIS BAYKANT ALAGOZ received bachelor degree in Istanbul Technical University, Department of Electronics and Communication Engineering in 1998, M.Sc. and Ph.D. degrees in Inonu University, Department of Electrical-Electronics Engineering in 2011 and 2015. His research interests include modeling and simulation of physical systems, control systems, systems and intelligence, smart grid. $\mathrm{He}$ is working at Computer Engineering Department in Inonu University.

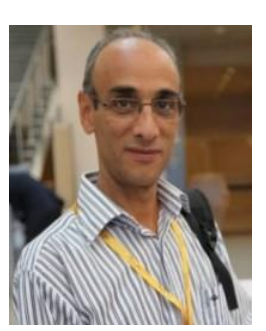

HAFIZ Z. ALİSOY, was graduated from Moscow Technical University department of ElectroPhysics Engineering 1982. He had his PhD degree from USSR Science Academy Physics Institute of P.N. Lebedyev and Doctor of Sciences degree (DSc) from International Ecology-Energy Academy. He became as Full Professor in 1995. He received award of Young Scientist. He works at Namık Kemal University, Department of Electronics and Telecommunication Engineering. 